\title{
Zwei Wege als Grund der vierteiligen Struktur von Parmenides' Gedicht: Entwurf eines neuen Ansatzes
}

\author{
Pavel Hobza
}

Bevor man Parmenides' Gedicht zu interpretieren anfängt, macht man eine wichtige interpretatorische Entscheidung, ohne es eigentlich innezuwerden. Man setzt nämlich voraus, dass Parmenides' Gedicht aus drei Teilen besteht. Obwohl man diese Voraussetzung fast für eine Tatsache hält, zeigt es sich bei näherem Zusehen, dass die Einteilung des Gedichts in drei Teile nur auf der Interpretation zweier Stellen beruht (auf einem bestimmten Verständnis der Beziehung zwischen den Fragmenten B 1 und B 2 und auf der Ergänzung der Lacuna am Ende von B 6,3). Und weil bei der Interpretation beider Stellen die Wegeproblematik eine wichtige Rolle spielt, zeigt es sich weiter, dass die Einteilung des Gedichts in drei Teile mit der Zahl der Wege resp. mit der gewöhnlichen Annahme dreier Wege weitgehend zusammenhängt. Eine der wichtigsten Aufgaben der vorgelegten Studie ist also eine alternative Struktur des Gedichts zu entwerfen und zu zeigen, dass das Gedicht anhand von zwei Wegen in vier Teile eingeteilt werden kann, ja muss.

Unsere Untersuchung gliedert sich in drei Hauptteile: Da die gewöhnlich angenommene dreiteilige Struktur mit der angeblichen ontologischen Argumentation des Parmenides zusammenhängt, setzen 
wir uns im ersten Hauptteil mit der geläufigen Art und Weise, wie man દ́óv in Parmanides' Gedicht erklärt, auseinander. Da diese Erklärung meistens auf die Behauptung hinausläuft, dass Parmenides die Ontologie begründet hat, und da die angebliche Parmenideische Ontologie auf der

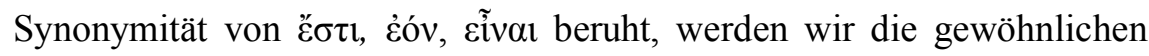
Interpretationen als ontologisch bezeichnen. Während aber die Ontologie mit einigen, in Parmenides' Text sowie im damaligen Kontext kaum nachweisbaren abstrakten Denkoperationen und Philosophemen ${ }^{\mathrm{i}}$ arbeiten muss, sollte man vielmehr versuchen, \&̇óv in Einklang mit den damaligen Denkmöglichkeiten zu erklären. Auch die gewöhnliche Gliederung des Gedichts in drei Teile ist - wie wir sehen werden - durch das ontologische Vorverständnis in hohem Maße gerechtfertigt. Im zweiten Hauptteil versuchen wir, eine neue, vierteilige Struktur des Gedichts, die auf der Annahme zweier Wege beruht, zu entwerfen. Der Vorteil dieser Struktur besteht unter anderem darin, dass sie dem Sachverhalt, dass Parmenides' Text ein der archaischen Kultur entstammendes, orales Gedicht ist, Rechnung trägt. Im dritten Hauptteil beschäftigen wir uns letztlich mit einzelnen spezifischen Problemen, die bei der Interpretation des Gedichts erscheinen und die meistens die Gestalt und Bedeutung des

\footnotetext{
i Vgl. z. B. Furth, M., „Elements of Eleatic Ontology“, in: A. P. D. Mourelatos (ed.), The Pre-Socratic: a collection of critical essays, Princeton, New Jersey 1993, S. 241-270, S. 241. "Several portions of the general view from which I 'deduce the poem' are not clearly stated in the poem itself; my explanation for this is that they are operating as tacit assumptions". Unter "tacit assumptions" versteht er abstrakte und ontologische Denkschemata. Dagegen vgl. Havelock, E. A., Preface to Plato, Oxford 1963, VII-VIII. "But if the early Greek mentality was neither metaphysical nor abstract, what then was it, and what was it trying to say? [...] The Presocratics themselves were essentially oral thinkers, prophets of the concrete linked by long habit to the past, and to forms of expression which were also forms of experience, but they were trying to devise a vocabulary and syntax for a new future, when thought should be expressed in categories organized in a syntax suitable to abstract statement."
} 
neuen zweiten „Wegeteils“ (der sich von B 1,24 bis zu B 7 erstreckt) betreffen.

\section{I.1 Angebliche Ontologie}

Wie oben schon angedeutet wurde, trägt man die Ontologie an Parmenides' Text heran, um den Ursprung, ja überhaupt die Möglichkeit von Éóv zu erklären. Im allgemeinen lässt sich sagen, dass man im Gedicht des Parmenides zwei Arten der ontologischen Argumentation entdeckt:

1) Parmenides soll bei der Begründung der Ontologie von dem als „Mittel der Untersuchung“ dienenden ̌ $\sigma \tau \iota$ ausgegangen sein (vgl. „Der Baum ist“ usw.). Weiter soll er zur Ansicht, dass

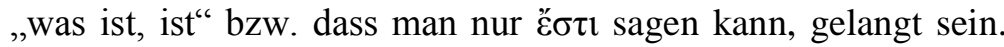

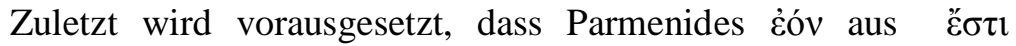
deduziert hat. ${ }^{\mathrm{i}}$

i Vgl. z. B.: Furth, M., „Elements of Eleatic Ontology“, op. cit., S. 261. “By an 'enquiry” into 'what is' I shall understand Parmenides to mean any investigation of 'what is' in the sense of what is so, or what is the case; any procedure aimed at ascertaining the facts." Ibid, S. 249. "What is (everything that is), is [...] and (very emphatically) that's all (=nothing else!)." Ibid, S. 264. "Parmenides' own ontology and cosmology, upon which attention has traditionally focused, and which I take it is agreed to be absurd, can all be derived, without mistakes, from the standpoint at which we have now arrived." Kahn, Ch. H., ,The Thesis of Parmenides“. The Review of Metaphysics 22, 1968/69, S. 700-724, S. 700-1. "I am primarily concerned here to elucidate Parmenides' thesis: to see what he meant by the philosophic claim which is compressed into the one-word sentence है $\sigma \tau 1$, 'it is.' I take this to be the premise (or one of them), from which he derives his famous denial of all change and plurality." Kirk, G. S., Raven, J. E., The Presocratic Philosophers, Cambridge 1982, S. 272. "The premise है $\sigma \tau \iota$ is by now established as the only possibility: the only significant thought or statement is that a thing is. [...] From now onwards until the end of the Way of Truth he is concerned, in other words, to deduce all that can be 
2) Man nimmt an, dass durch ह̌ $\sigma \tau \imath$, das hier unpersönlich und existential verwendet werden soll, das Sein oder die

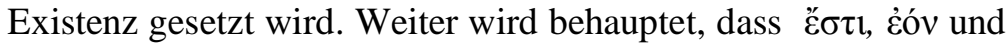
Eĩval (anhand eines reinen Seinsbegriffs) einfach synonym sind. ${ }^{\mathrm{i}}$

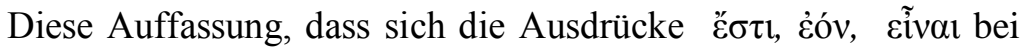
Parmenides aufeinander beziehen, einander übertragbar oder sogar auf einen (Seins-)Begriff zurückzuführen sind (sei es anhand der Deduktion oder der Synonymität), scheint aber im Text des Parmenides, geschweige denn im damaligen Kontext, kaum Unterstützung zu finden; denn sie setzt einige, erst später bei Platon und Aristoteles anzutreffende Philosopheme voraus. Wenn man also die Ontologie bei Parmenides zu rekonstruieren versucht, stößt man auf eine im Gedicht wohl nie wirklich befriedigend

deduced from his chosen premise about the properties of Being.” Tugendhat, E., „Das Sein und das Nichts“, in: E. Tugendhat, Philosophische Aufsätze, Frankfurt a. M. 1992, S. 36-66, S. 38. „Wie hat Parmenides, der erste Philosoph, der auf das ,ist“-Sagen aufmerksam wurde, das Wort ,ist` verstanden? Aus der richtigen Antwort auf diese Frage muss verständlich werden, wieso Parmenides meinen konnte, dass aus dem Sinn des ,ist " und seiner Entgegensetzung zum ,ist nicht' notwendig folgt, dass, was ist, nur eines sein kann, notwendig unvergänglich, unveränderlich und homogen." Ähnlich auch Heitsch, E., Parmenides. Die Anfänge der Ontologie, Logik und Naturwissenschaft, München 1974. Der Unterschied besteht nur darin, dass er statt von ह̌ $\sigma \tau \imath$ von der

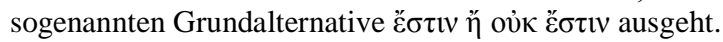

i Vgl. z. B.: Cordero, N.-L., Les deux chemins de Parménide, Paris 1984, S. 74-75. „Lorsque Parménide dit ,esti ${ }^{6}$, il constate (ou propose) un fait: qu'il y a, qu' existe, que quelque chose est présant. [...] Parménide part d'une thèse [...]: la présance, l'existance, le fait d'etre. Ce principe, Parménide l'exprime indifféremment moyennant un infinitif

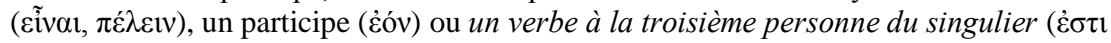
comme en 2,3a et en 2,5a." Taran, L., Parmenides. A Text with Translation, Commentary, and Critical Essays, Princeton, New Jersey 1965, S. 37. „let it be stated once and for all that the different idioms which Parmenides uses to express Being and non-Being are synonymous. [...] To distinguish between the use of the participle and of the infinitive, for example, as some scholars do, is to obliterate the fact that for Parmenides there is only absolute Being, although the language, the meter, and the necessity of referring to the phenomenal world in order to deny its existence forced him sometimes to use expressions like ,the things which are not" (fr. VII.1)." Vgl. auch Wiesner, J., Parmenides. Der Beginn der Aletheia, Berlin - New York 1996, S. 112-122. 
aufzulösende Spannung zwischen der Konzeption von ह̌ $\sigma \tau \iota$, die mit einem rein geistigen Akt oder Begriff des Seins zu rechnen hat, und der von દ̇óv, die mit räumlichen Prädikaten und Vorstellungen verbunden ist (vgl. Charakterisierungen von દ̇óv im Fragment B 8 wie z.B. seine Unteilbarkeit in B 8,22-25 oder seine Kugelförmigkeit und Begrenztheit in B 8,42-49) - eine Spannung, die erst anhand des weder innerhalb der archaischen Kultur, noch innerhalb der vorsokratischen Philosophie nachweisbaren Philosophems Materialität versus Immaterialität verständlich und möglich wäre. Wenn man dieses Phlilosophem im Gedicht irgendwie voraussetzt, dann fragt es sich etwa, warum Parmenides - wenn er einmal den immateriellen Begriff des Seins im Ě $\sigma \tau$ gefunden hatte - ihn dann in der Konzeption von દ̇óv mit räumlichmateriellen Prädikaten versah; warum er also das Materielle und das Immaterielle vermischt und nicht auseinanderhält. Man kann sicher erwidern: eben deshalb, weil er dieses Philosophem nicht gehandhabt hat und weil ,he may have been attempting to conceive a nonspatial Reality but was simply unable to find any expression for this view except in spatial language.",

Man scheint also den Schwierigkeiten, die mit der Formulierung und Begründung der Ontologie im Gedicht zusammenhängen, durch die Behauptung zu entgehen, dass es Parmenides nicht der geeignete begriffliche Apparat zur Verfügung stand. Doch im Allgemeinen lässt sich sagen, dass die ontologische Interpretation einerseits mit sehr

i Kahn, Ch. H., „Parmenides, ed. Tarán“, Gnomon 40, 1968, S. 132. Ähnlich auch Kirk, G. S., Raven, J. E., The Presocratic Philosophers, op. cit., S. 270. "On the contrary, the chief difficulty about Parmenides is that, while the incorporeal was still unknown, and no vocabulary therefore existed to describe it, he was none the less [...] feeling his way towards it." 
gewagten, andererseits mit sehr vagen Voraussetzungen und Vorstellungen arbeitet; weshalb es angebracht ist, nach einer anderen, der damaligen Zeit angemesseneren Erklärung von çóv zu suchen, als sie die

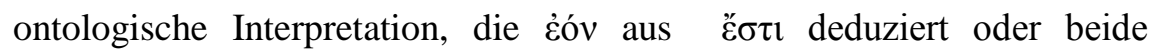
Termini einfach als synonym betrachtet, liefert.

\section{I.2 Der Ursprung von żóv}

Es ist viel plausibler, દ́óv aus dem damals geläufigen Ausdruck દ̇óv $\tau \alpha$, der die Welt im ganzen bezeichnet hat, zu erklären. Diese Erklärung ist hauptsächlich aus zwei Gründen der ontologischen vorzuziehen:

Erstens ist sie den damaligen Denk- und Sprachmöglichkeiten angemessen. Man braucht also nicht vorauszusetzen, dass Parmenides von einer Art ontologischer Spekulation, die sich - wie wir gesehen haben - einiger komplizierter und abstrakter Denkoperationen oder Philosopheme bedienen muss, ausgegangen sei. Er scheint vielmehr

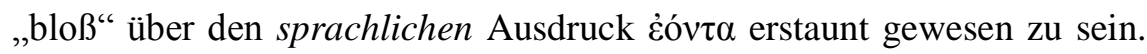
Dass man über die Welt als über દ̇óv $\tau \alpha$ sprechen kann, impliziert, dass jedes einzelne Ding als \&́óv begriffen werden kann. Parmenides muss sich also die (geniale) Frage gestellt haben: Wie können sich alle Dinge voneinander unterscheiden, wenn jedes Ding letztlich als ein einzelnes દóv aufgefasst werden kann? Wobei seine Antwort gelautet haben muss: Sie unterscheiden sich gar nicht, weswegen es letztlich nur ein einziges દ̇óv gibt. In anderen Worten, beim Konzipieren von દ̇óv scheinen Parmenides' Erwägungen über die diskreten Seienden eine wichtige Rolle 
gespielt zu haben. Obwohl dieser Gedankengang ganz und gar ontologisch anmuten kann, ist der von uns angenommene Ursprung von દ́óv in einer wichtigen Hinsicht von der ontologischen Argumentation verschieden. Denn gegenüber der geläufigen ontologischen Interpretation meinen wir nicht, dass Parmenides imstande war, einzelne in der Welt sich befindende Dinge als die Seienden deshalb aufzufassen, weil sie sind (d.h. weil ihnen das Prädikat ,ist“ zugeschrieben werden kann). Dieser Gedanke kann zwar für uns ganz und gar selbstverständlich sein, aber in Parmenides' Text, geschweige denn im damaligen Kontext findet sie keine Stütze. Deshalb scheint es uns den damaligen Denkmöglichkeiten angemessener, den Ursprung von દóv bloß in einem sprachlichen Ausdruck resp. in Parmenides' Erwägungen über die Möglichkeiten und Tragweite dieses Ausdrucks zu sehen. (Obwohl es für uns kaum vorstellbar ist, dass anhand der Sprache auf die Realität geschlossen werden kann, ist dieses Schließen in Rahmen der archaischen Denkweise durchaus plausible. Denn damals wurde noch nicht die strikte und unüberbrückbare Trennung zwischen der Sprache und Realität vollzogen, so dass beide als Aspekte des Gleichen angesehen werden konnten.)

Die Richtigkeit dieses Ursprungs von çóv wird aber im Gegensatz zur ontologischen Erklärung, die erst anhand einiger, bei Parmenides nirgendwo explizit nachweisbarer Voraussetzungen gerechtfertig werden kann, durch eine deutliche Spur im Gedicht bestätigt. Es handelt sich um

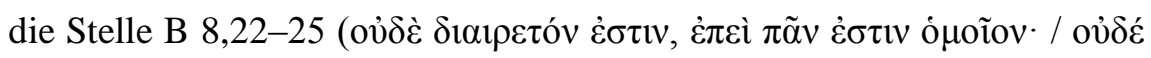

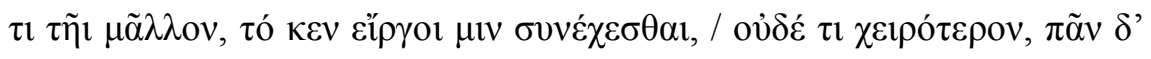

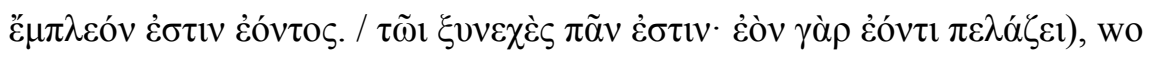
die (Unmöglichkeit der) Teilbarkeit von غ̇óv erörtert wird und wo

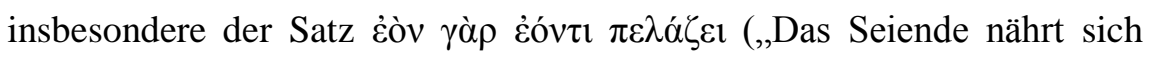


dem Seienden“, was in dem Sinn zu verstehen ist, dass das Seiende dem Seienden ähnlich ist) hervorzuheben ist. Denn im Unterschied zur ontologischen Interpretation wird es hier angedeutet, dass die Konzeption von ćóv irgendwie mit Parmenides' Überlegungen über die diskreten Seienden, die sich letztlich ähneln, zusammenzuhängen scheint (vgl. auch

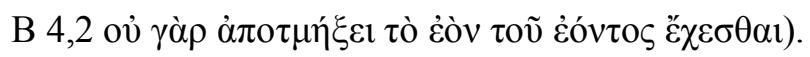

Der von uns angenommene Ursprung von ćóv scheint aber zweitens die Erklärung der Beziehung zwischen દ̇óv und der ab B 8,53 behandelten menschlichen Welt anzudeuten, was für die meisten Interpretationen bekanntlich das schwierigste Problem ist. Obwohl sie oft nicht imstande sind, die Möglichkeit der menschlichen Welt auf dem Hintergrund von Éóv einzusehen, ${ }^{\text {i }}$ sollte man schon anhand der Tatsache, dass Parmenides der Beschreibung der menschlichen Welt soviel Raum und Mühe gewidmet hat (der weitaus größte Teil des Gedichts war ja der Beschreibung der Welt vorbehalten), voraussetzen, dass die Welt im Gedicht eine durchaus wichtige Rolle spielen muss und dass Parmenides

\footnotetext{
i Vgl. z. B. Mansfelds resignierte Äußerung, dass die Frage, ,wie Parmenides es verantworten kann, dass neben (?) dem unbewegten und ewigen Seienden noch eine menschliche Welt entstehen kann, [...] nicht mit vollständiger Gewissheit beantwortet werden“" kann (Mansfeld, J., Die Offenbarung des Parmenides und die menschliche Welt, op. cit., S. 219), was dann Kahn in der Besprechung Mansfelds Buch mit „Plotinus’ question, "Why is there more than the One'?" vergleicht und was er als "the problem which remains, and must remain, unresolved” auffasst (Kahn, Ch. H., „Jaap Mansfeld: Die Offenbarung des Parmenides und die menschliche Welt", Gnomon 42, 1970, S. 119). Man sollte aber erwägen, ob eine Interpretation, die bei solchen (sei es durch die Resignation oder durch den Hinweis auf eine entlegene Tradition zu rechtfertigenden) Ausflüchten Zuflucht sucht, eine wirklich gelungene Interpretation ist. Man sollte daher weiter bedenken, ob nicht gerade eine befriedigende, im Text plausibel nachweisbare und womöglich mittels der damaligen Denkmöglichkeiten durchgeführte Lösung dieser Beziehung ein wichtiges Kriterium für die richtige Interpretation lieferte; man scheint nämlich bisher ein solches Kriterium vor allem im Nachweis der ontologischen Argumentation zu sehen.
} 
sie nicht einfach verwerfen und durch die Konzeption von દ̇óv

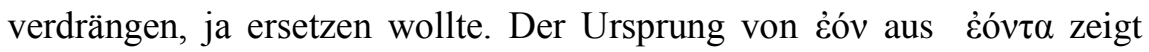

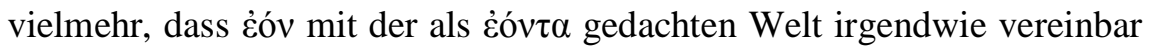
sein und sie voraussetzen muss. ${ }^{\mathrm{i}}$ Wie die Beziehung zwischen غ̇óv und der als ċóv $\tau \alpha$ begriffenen Welt innerhalb der Lehre des Parmenides genau aussieht, kann aber erst später (vgl. Abschnitte III.3, 4.) behandelt werden.

Diese Ausführungen haben für die angebliche parmenideische Ontologie erhebliche Konsequenzen; denn damit ist die enge (sei es anhand der Deduktion oder der Synonymität) angenommene Beziehung

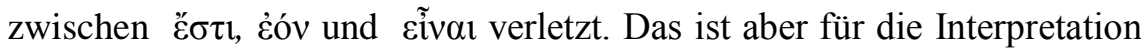
des Gedichts von großer Bedeutung, weil man damit etwa die

i Im Wesentlichen ist also der Interpretation Schwabls zuzustimmen, dass die menschliche Welt positiv zu bewerten ist und dass die Welt oder die sie konstituierenden Kräfte: Licht und Nacht irgendwie im śóv enthalten sind oder aufgehoben werden. Seine Auffassung, „dass Licht wie Nacht ,sind““ (Schwabl, H., „Sein und Doxa bei Parmenides“, in: H.-G. Gadamer (ed.) Um die Begriffswelt der Vorsokratiker, Darmstadt 1989, S. 391-422, S. 396) und dass sich anhand dieses ,es ist“ im Sein als aufgehoben und vereint erweisen, muss jedoch korrigiert werden. Denn Licht und Nacht können im દ́óv aufgehoben werden, weil sie (sprachlich) als ćóv $\tau \alpha$ zu verstehen sind.

Dass die menschliche Welt mit દ̉óv im Grunde gleichzusetzen ist, könnte im Text noch durch zweierlei gestützt werden. Erstens: In B 8,5 und B 8,22-25 wird غ̇óv als ein Ganzes $(\pi \tilde{\alpha} v)$ verstanden; in B 9,3 befindet sich dieselbe Bezeichnung für die aus Licht und Nacht bestehende Welt. Im Hinblick auf $\pi \tilde{\alpha} v$ scheinen ćóv und die menschliche Welt dasselbe zu sein. Zweitens durch folgende Interpretation von B 1,31-32: „Du wirst aber auch das erfahren, dass die erscheinenden Dinge ( $(\pi \alpha ́ v \tau \alpha)$ durch das Ganze $(\pi \tilde{\alpha} v)$ durchgehen." Dass Parmenides hier zwei sprachlich

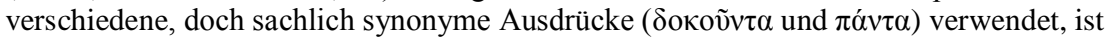

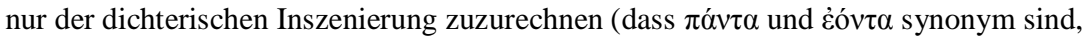

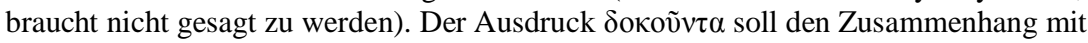

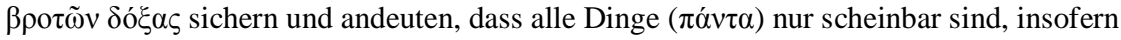
sie nicht im દ̇óv resp. $\pi \tilde{\alpha} v$ (ein)begriffen sind. - Vgl. dazu auch Steigers Konzeption des geschlossenen kosmologischen Schemas (Steiger, K., „Die Kosmologie des Parmenides und Empedokles“, Oikumene 5, 1986, S. 173-236). 


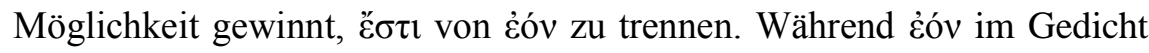
als der zu behandelnde Gegenstand der Untersuchung auftritt, hat ह̌ $\sigma \tau \imath$ vornehmlich die Funktion eines geläufigen Prädikats.

\section{II.1 Alternative Gliederung des Gedichts}

Das Gedicht wird herkömmlicherweise in drei Teile: Proömium, aletheia- und doxa-Teil eingeteilt. Die Einteilung zwischen aletheia- und doxa-Teil bereitet keine Schwierigkeiten, weil beide Teile durch B 8,50-

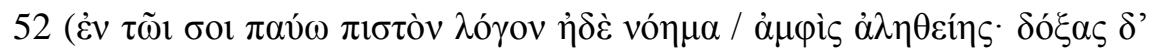

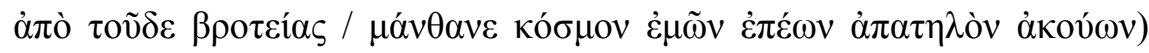
deutlich und eindeutig abgetrennt sind. Man kann sich aber fragen, wie die gewöhnlich angenommene Einteilung zwischen Proömium (das als Fragment B1 verstanden wird) und aletheia-Teil (B2-B8) zu rechtfertigen ist, denn zwischen ihnen gibt es keine klare stilistische Abgrenzung.

Darin, dass man zwischen B1 und B2 eine so scharfe Abgrenzung anzunehmen pflegt, scheinen hauptsächlich zwei Gründe mitzuspielen: Der eine Grund darf auf unserer bruchstückhaften Überlieferung des Gedichts beruhen. Denn das Proömium wird einfach, ohne dass man sich darüber viel Bedenken machte, mit dem Fragment B 1 gleichgesetzt; die scheinbare (bloß in unseren Ausgaben sich manifestierende) Selbstständigkeit von B 1 erweckt also den Eindruck der inneren Geschlossenheit. Das scheint mit dem anderen, in dem ontologischen Vorverständnis bestehenden Grund in völligem Einklang zu stehen. Denn in B 1 scheint nichts philosophisch Wertvolles, d.h. Ontologisches zur Sprache zu kommen; da handelt es sich ja lediglich um 
die bildliche Schilderung einer Fahrt und in den letzten Versen wird einiges über die Gliederung des Gedichts in zwei Teile angedeutet. Erst in B 2 setzen - wie man annimmt - die eigentlichen philosophischontologischen Ausführungen ein.

Mit der Einteilung zwischen das als Fragment B 1 verstandene Proömium und den in B 2 einsetzenden aletheia-Teil hängt aber unmittelbar die gewöhnliche Annahme, dass es im Gedicht drei Wege gibt. Man nimmt nämlich an, dass die in B 1,29-30 vorkommende

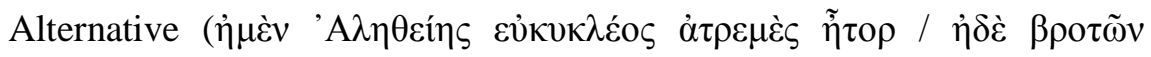

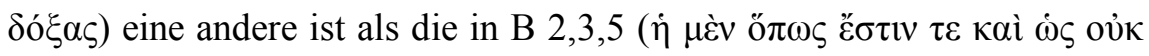

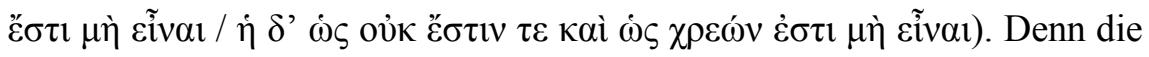
erste Alternative scheint über das Sein und die menschliche Welt zu sein, während die zweite über das Sein und Nichtsein. Man nimmt also an, dass die erste Alternative im allgemeinen mit der Einteilung des Gedichts in aletheia- und doxa-Teil zusammenhängt, während die zweite eine logisch-ontologische Alternative zwischen dem Sein und Nichtsein darstellt. Und weil das erste Glied beider Alternativen denselben Gegenstand, d.h. das Sein, impliziert und deshalb dasselbe sein muss, nimmt man an, dass es im Gedicht drei Wege gibt. ${ }^{\mathrm{i}}$

Nun, diese Auffassung, dass beide Alternativen verschieden sind, kann - bisher nur vorläufig - problematisiert werden, womit aber gleich die Annahme dreier Wege problematisiert wird. Denn wenn wir erwägen, dass B 2 ursprünglich in unmittelbarer Nähe zu B 1 gestanden haben

i Obwohl die erste Alternative die Wege nicht explizit thematisiert, man nimmt an, dass auch sie die Wege betrifft. 
muss (es ist ja sogar anzunehmen, dass es an B 1 unmittelbar anschloss ${ }^{\mathrm{i}}$ ), dann ist es nicht ohne weiteres verständlich, dass die in B 1,29-30

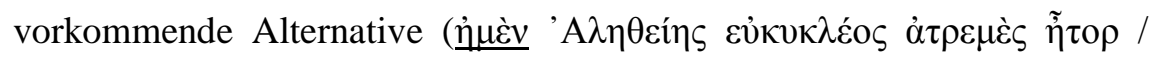

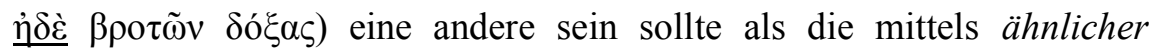

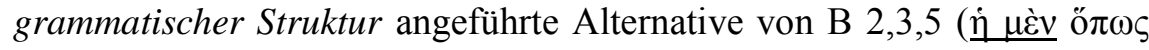

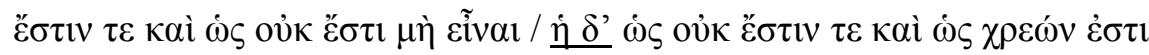
$\mu \eta \grave{~ \varepsilon i ̃ v a l) . ~ W e n n ~ w i r ~ w e i t e r ~ i n ~ B e t r a c h t ~ z i e h e n, ~ d a s s ~ w i r ~ e i n ~ d e r ~}$ archaischen Kultur entstammendes Gedicht, das wohl nicht zum (einsamen und lautlos verstehenden) Lesen, sondern zum (öffentlichen und lauten) Vortragen bestimmt wurde, interpretieren, dann wäre es wieder vorläufig - anzunehmen, dass beide Alternativen vielmehr dieselben sind. Denn damit das Vorgetragene von Zuhörern leicht und mit Verstehen verfolgt werden kann, bedienen sich die oralen, zum Vortragen bestimmten Texte der Wiederholung als eines wichtigen stilistischen, ja kompositorischen Mittels. Deshalb würde man erwarten, dass beide Alternativen nicht verschieden sind, sondern vielmehr eine Wiederholung bzw. eine wiederholte Variante einer und derselben Alternative darstellen.

Weiteres Problem der gewöhnlichen Einteilung des Gedichts in drei Teile liegt darin, dass einige Themen vom einen Teil ins andere unbegründet übergreifen. So sollte der aletheia-Teil die Wahrheit resp. die mit der Ontologie oder mit dem Sein zusammenhängenden Probleme im Gegensatz zu menschlichen Meinungen, denen ja der doxa-Teil vorbehalten ist, behandeln. Obwohl dies B 2 durchaus zu bestätigen scheint, fragt es sich, warum in B 6 und B 7 der Weg der schweifenden Menschen thematisiert wird. Denn damit scheint nicht nur der doxa-Teil

\footnotetext{
i Vgl. Heitsch, E., Parmenides. Die Anfänge der Ontologie, Logik und Naturwissenschaft, op. cit., S. 140 .
} 
vorgegriffen, sondern auch das Thema von B 1,26-30 aufgegriffen zu werden, wo über den menschlichen Weg im Gegensatz zu dem, den $\theta \varepsilon \dot{\mu} \iota \varsigma$

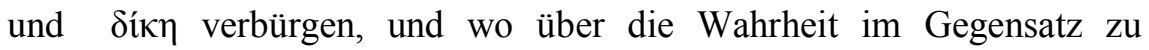
menschlichen Meinungen die Rede ist.

Wenn aber die gewöhnliche Einteilung des Gedichts in drei Teile solche Probleme impliziert, können wir uns - mindestens vorläufig und versuchsweise - vorzustellen versuchen, wie das Gedicht alternativ eingeteilt werden könnte. Wie wir gesehen haben, ist die Einteilung von B 8,50-52 unproblematisch. Weil aber die Einteilung zwischen B 1 und B 2 problematisch zu sein scheint, sind andere eindeutigere Abgrenzungen im Gedicht zu suchen. Wenn wir das Gedicht unbefangen übersehen, könnten wir zwei bisher noch nie berücksichtigten Abgrenzungen finden. Die erste sehr wichtige und deutliche Einteilung ist da zu setzen, wo die Göttin zu sprechen anfängt, also in B 1,24. Wenn wir in Betracht ziehen, dass das Gedicht als der Vortrag der Göttin, dem eine kurze bildliche Schilderung der Auffahrt des Parmenides (als des Jünglings, vgl. die Anrede der Göttin in B 1,24 кои̃ $\rho$ ) vorausgeht, konzipiert ist, dann gibt es keinen Grund, warum dieser deutliche und klare Einschnitt im Gedicht nichts zu seiner Gliederung beitragen sollte. Das Proömium wäre also nicht mit dem ganzen Fragment B 1 gleichzusetzen, wie man es gewöhnlich tut. Das Proömium würde nur die Verse B 1,1-23 enthalten, in denen die Auffahrt geschildert ist, wobei der Rest von B 1, wo die Göttin selbst schon $\mathrm{zu}$ sprechen angefangen hat, einen anderen Teil darstellt. Die andere ziemlich deutliche stilistische Abgrenzung findet sich am Anfang von B 8, wo gesagt wird, dass noch ein Wort über den Weg, der durch die Redewendung $\dot{\omega} \varsigma$ ह̌ $\sigma \tau \imath v$ gekennzeichnet ist ( $\mu$ óvo $\delta$ '

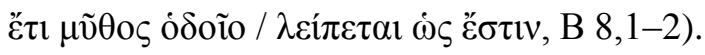


Wenn es also im Gedicht (resp. in den erhaltenen Fragmenten) drei deutliche Einschnitte oder Abgrenzungen gibt, muss das Gedicht aus vier (und nicht - wie man bisher angenommen hat - aus drei) Teilen bestehen. Wenn wir weiter erwägen, dass der zweite Teil von B 1,24, wo die Göttin zu sprechen anfängt, bis zum Anfang von B 8 erstreckt (und dass also die Alternativen von B 1,29-30 und von B 2 innerhalb desselben Teils vorkommen), dann wird auch die gewöhnliche Annahme dreier Wege fragwürdig. Denn es wäre einfacher, ja eleganter, im Gedicht nur zwei Wege anzunehmen.

Bevor wir aber versuchen, das Gedicht anhand zweier Wege in vier Teile zu strukturieren, ziehen wir noch einmal in Betracht, dass wir ein orales, d.h. für das laute und öffentliche Vortragen bestimmtes Gedicht interpretieren. Das kann insofern von Bedeutung sein, als mit den Erwartungen und überhaupt Verstehensmöglichkeiten des Zuhörers zu rechnen ist. Um von Zuhörern leicht und mit Verstehen verfolgt werden zu können, sollte das orale Gedicht eine klare und übersichtliche Struktur haben. Was die gewöhnliche Einteilung des Gedichts in drei Teile unter der Voraussetzung dreier Wege angeht, wirkt die so aufgefasste Struktur eher verwirrt. Denn einige Gedanken, die in einem Teil ihren rechten Platz zu haben scheinen, tauchen in einem anderen auf, wo sie einigermaßen ungeeignet wirken (z.B. das mit den schweifenden Menschen zusammenhängende Motiv des Wegs, das in dem für die Behandlung der ontologischen Probleme bestimmten aletheia-Teil vorkommt). Überdies wäre der angebliche Verweis auf die Einteilung des Gedichts, der am Ende des als Proömium verstandenen Fragment B 1 erscheint und nach dem das Gedicht in zwei Teile strukturiert werden soll, vielmehr in einem schriftlichen, für das einsame verstehende Lesen 
bestimmten Text zu erwarten. Denn während man bei Lesern damit rechnen kann, dass sie sich auch anhand des wiederholten Lesens den schriftlichen Text anzueignen und seinen Sinn und Struktur kennen zu lernen versuchen, muss sich der Zuhörer nur auf einen Vortrag verlassen; deshalb bedient sich der orale Text auch anderer Mittel der Strukturierung (z.B. des Wiederholens desselben Motivs). In anderen Worten, es ist nicht $\mathrm{zu}$ erwarten, dass man den oralen Text nur mittels einer anfänglichen Erwähnung in zwei komplexe und weiter sich gliedernde Teile strukturiert (hauptsächlich wenn diese Struktur nur angedeutet wird).

Versuchen wir nun also die einzelnen Teile kurz zu characterisieren:

Den ersten Teil bildet das Proömium, das aber nicht - wie man gewöhnlich annimmt - am Ende vom Fragment B 1, sondern da, wo die Göttin zu sprechen anfängt, also in B 1,23, endet. Das Proömium, das eine bildliche Schilderung der Auffahrt darstellt, ist nach der am Bild des Übergangs von der Nacht zum Licht inszenierten binären Struktur aufgebaut (die Sonnenmädchen verlassen das Haus der Nacht und streben zum Licht; sie stoßen die Schleier von ihren Häuptern weg; weiter

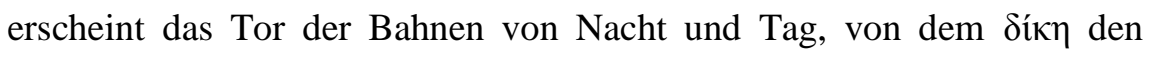
Schlüssel hat; Parmenides passiert dieses Tor und wird von der Göttin in ihrem Haus, das als Gegensatz zum Haus der Nacht konstruiert wird, empfangen).

Was die Zahl der Wege im Gedicht angeht, wäre es überraschend, wenn die deutliche binäre Struktur, nach der das ganze Proömium aufgebaut ist und die bereits im Proömium als Motiv zweier Wege von

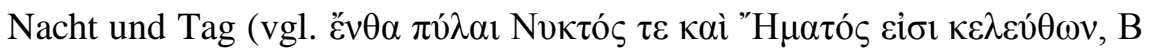


1,11) vorgezeichnet ist, in den weiteren Darlegungen als Motiv zweier Wege nicht ausgewertet würde. Dieselbe binäre Struktur kommt also -

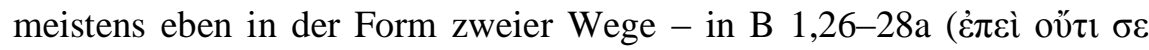

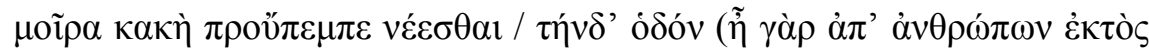

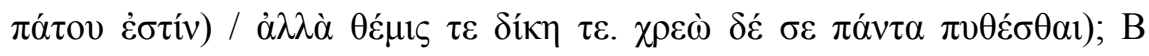

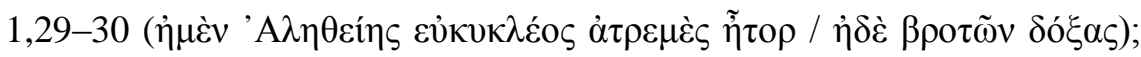

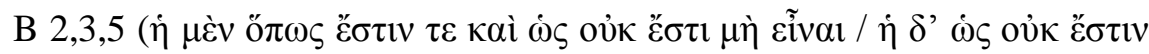

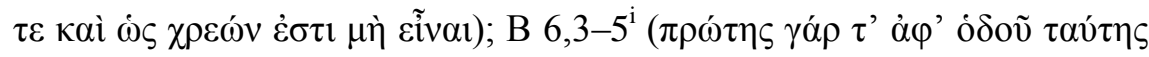

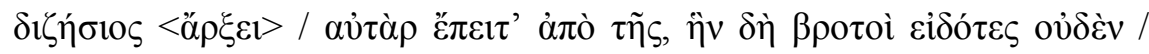

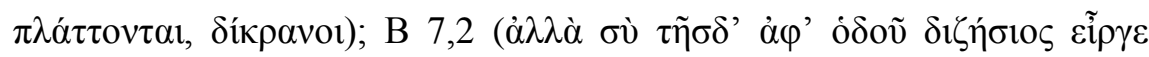

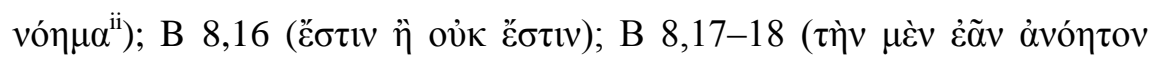

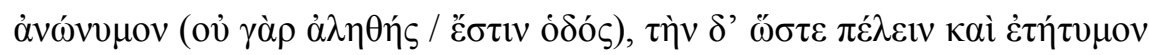
Eĩval) vor. Die zwei Wege stellen also das Grundmotiv des Gedichts, anhand dessen das Gedicht durchaus streng eingeteilt und strukturiert ist, dar.

i Die Interpretation dieser Verse stellt die zweite Stelle, die (außer der angeblichen, auf dem ontologischen Vorverständnis gründenden Verschiedenheit von B 1 und B 2) für die Annahme dreier Wege resp. dreier Gedichtsteile zu sprechen scheint, dar. Am Ende des Verses B 6,3 fehlt bekanntlich ein zweisilbiges Wort, das von Diels durch Elp $\gamma \omega$ (,,ich halte zurück") ergänzt wurde. Seine Ergänzung wurde seitdem fast einstimmig akzeptiert. Dass jedoch das in B 6,3 von Diels ergänzte ěp $\gamma \omega$ durchaus willkürlich ist und dass man da vielmehr $\not \alpha \rho \xi \varepsilon 1$ (,fange an“) ergänzen sollte, scheint uns von Cordero überzeugend nachwiesen worden zu sein (vgl. Cordero, N.-L., Les deux chemins de Parménide, op. cit., S. 110-175). Und wie Cordero weiter argumentiert, hängt die Zahl der Wege im Gedicht (ob sie zwei oder drei sind) eben von dem in der Lacuna gewählten Wort ab. Wenn man da ő $\rho \xi \varepsilon ı$ ergänzt, heißt es, dass die Göttin auffordert, zuerst von dem einen, gerade beschriebenen Weg anzufangen, dann aber von dem, auf dem die unwissenden Sterblichen herumschweifen; in diesem Fall sind im Gedicht nur zwei Wege anzunehmen. Wenn man dagegen عíp $\gamma \omega$ ergänzt, heißt es, dass die Göttin von einem Weg zurückhält, dann aber von einem anderen. Weil sie von zwei Wegen zurückhält, muss es noch einen anderen positiven, d.h. dritten Weg geben.

ii Dass man von einem Weg zurückgehalten werden soll, impliziert, dass es noch einen anderen Weg, dem man sich zuwenden muss, gibt. 
Nachdem also das Motiv zweier Wege im Proömium vorgezeichnet war, wird es im zweiten Teil (ab B 1,24 bis B 7), der bisher noch nie beachtet und erkannt wurde, mehrmals wiederholt. Es handelt sich aber nicht um bloße Widerholung desselben Motivs, sondern vielmehr um seine Variierung resp. philosophische Ausarbeitung, die damit wir unsere weiteren Ausführungen ein bisschen vorwegnehmen dazu dient, beide Wege gegeneinander zu inszenieren und konfrontieren und so den Vorzug des ersten Wegs gegenüber dem zweiten nachzuweisen und $\mathrm{zu}$ sichern (vgl. Abschnitt III.3.). Dieses klare Wiederholen und Variieren desselben binären Motivs wird aber von den Interpreten ganz übersehen und das gleiche binäre Motiv wird in verschiedenen Teilen des Gedichts verschieden interpretiert resp. als verschiedene Alternativen gedeutet (vgl. z.B. oben angeführtes Beispiel von der in B 1,29-30 vorkommenden Alternative, die nach der geläufigen Auffassung von der in B 2 vorkommenden Alternative grundverschieden sein soll, was fast als Grundsatz der gewöhnlichen ontologischen Interpretation gilt). Es soll noch einmal erinnert werden, dass das Wiederholen und Variieren desselben Motivs eines der wichtigsten stilistischen, ja kompositorischen Mittel darstellt, denen sich ein oraler, d.h. für das Vorlesen und Vortragen bestimmter Text bedient.

Nun, wie wir noch sehen werden, ist die Wegeproblematik in Parmenides' Gedicht sehr komplex aufgefasst. An dieser Stelle ist es jedoch ein wichtiger Aspekt dieser Problematik herauszuarbeiten. Wie in B 1,29-30 angedeutet ist, hängt der eine Weg irgendwie mit ċóv zusammen, während der zweite mit der menschlichen Welt. Angesichts dessen, dass im Gedicht éóv und die Welt behandelt werden, ist ihre Beziehung zu beiden Wegen zu erwarten. Die Frage ist aber, wie diese 
Beziehung genau aussieht bzw. wie beide Wege zueinander stehen. Auf die genaue Beziehung beider Wege zu čóv resp. der Welt werden wir später eingehen (vgl. Abschnitt III.4.), deshalb versuchen wir jetzt vielmehr die Beziehung beider Wege zueinander verständlich machen. Denn nach der geläufigen ontologischen Auffassung, nach der sich દ́óv und die Welt gegenseitig ausschließen, wäre auch zwischen beiden mit દ̇óv resp. der Welt zusammenhängenden Wegen letztlich keine Beziehung; höchstens würde es sich um eine Beziehung der Ausschließung oder Privation handeln, was aber keine eigentliche Beziehung ist.

Wenn wir jedoch in Betracht ziehen, dass damals die Welt als દ̇óv $\tau \alpha$ aufgefasst wurde und dass - wie wir gesehen haben - Parmenides'

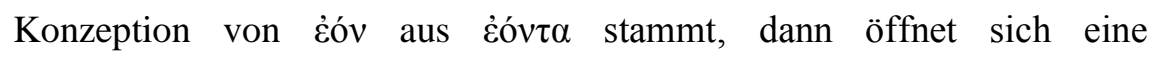
aussichtsvolle Möglichkeit, die Beziehung beider Wege zu denken. Die

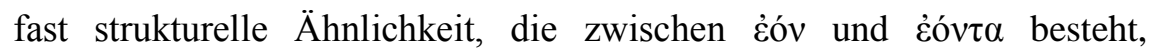
begründet auch die Beziehung beider Wege. Denn nicht nur dass sie sich nicht ausschließen, sondern wegen der Beziehung der mit ihnen

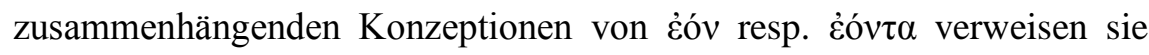
aufeinander, ja sie bedingen sich gegenseitig.

Man könnte vielleicht einwenden, dass die Wege im allgemeinen mit દ̇óv und der Welt zusammenhängen können, dass aber der zweite Weg nirgends explizit mit દ́óv $\tau \alpha$ verbunden ist. Denn die Welt resp. der mit ihr zusammenhängende Weg wird im Gedicht vielmehr mit dem Nichtsein (vgl. B 2,7; B 7,1-2) oder mit der Mischung der elementaren Grundkräfte von Tag und Nacht (z.B. B 8,53-59) verbunden. Dieser Einwand ist aber verfehlt, denn es wäre gleich zu behaupten, dass wegen 


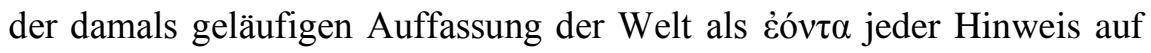

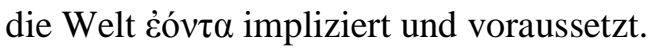

Unsere Auffassung von der Verbindung beider Wege mit çóv resp. Éóvta hat aber eine sehr wichtige Parallele, die sie durchaus bekräftigt. Es handelt sich um Empedokles' Fragment B 17, wo die Beziehung zwischen $\tilde{\varepsilon} v$ und $\pi \lambda \varepsilon \dot{o}$ v $\alpha$ behandelt wird. Denn Empedokles variiert und entfaltet hier die (dialektische) Beziehung zwischen $\check{\varepsilon} v$ und

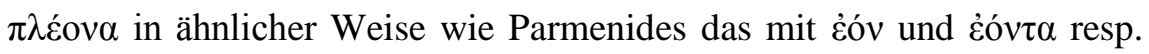
der Welt zusammenhängende Motiv zweier Wege. Es lässt sich erwarten, dass Empedokles, der ja in manchem an Parmenides anknüpft, auch dieses Motiv von ihm übernommen hat. Während jedoch Parmenides die

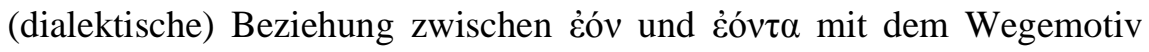
eng verflochten, ja in ihm aufgelöst hat (aus Gründen, die erst allmählich aufleuchten können und die - damit wir sie einigermaßen vorwegnehmen - mit dem praktisch-religiösen Charakter des Gedichts zusammenhängen, vgl. Abschnitte II.2. und III.5.), war Empedokles (wohl aus kosmogonisch-kosmologischen Gründen) darum bestrebt, eben die

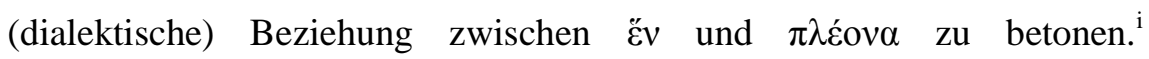

i Vgl. Empedokles DK 31 B 17,1-17:

$\delta i ́ \pi \lambda$ '

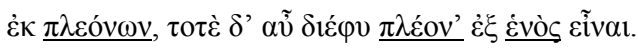

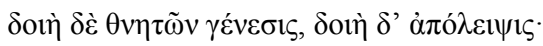

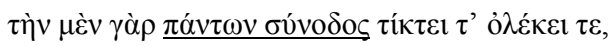

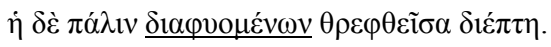


Empedokles' Fragment B 17 ist also für Parmenides' Gedicht insofern von Bedeutung, als man den zweiten (bisher nie erkannten) Wegeteil des Gedichts durch Empedokles' Fragment B 17 formal und inhaltlich gerechtfertig und bestätigt sehen kann.

Der dritte Teil des Gedichts beginnt wieder deutlich in B 8,1-2

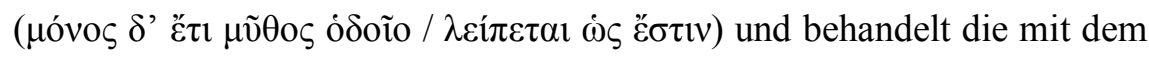
ersten Weg zusammenhängende Problematik von ćóv, während der vierte,

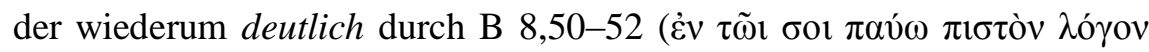

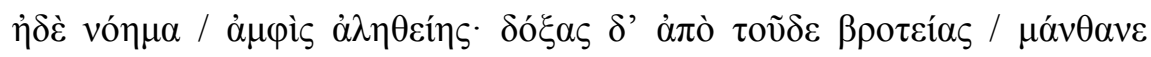

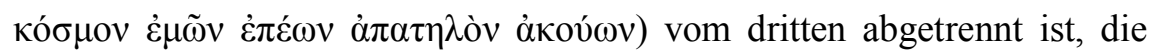

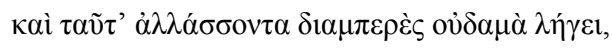

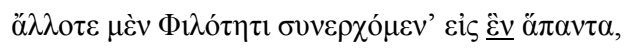

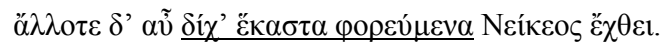

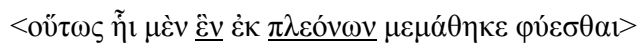

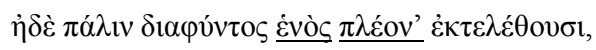

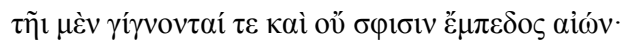

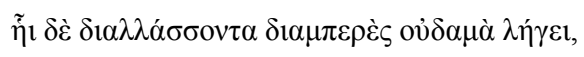

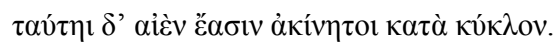

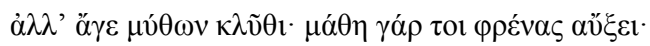

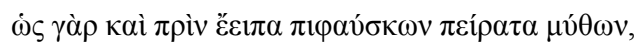

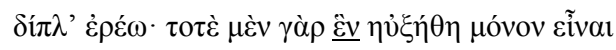

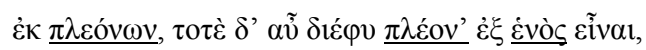


mit dem zweiten Weg zusammenhängende Problematik der menschlichen Welt.

\section{II.2 Archaische Anthropologie}

Wie Mansfeld anhand der Analyse von B 6 festgestellt hat, ist für Parmenides die archaische Auffassung des Menschen (die sogenannte archaische Anthropologie), die ihn als unwissend, hilflos, taub und blind im scharfen Gegensatz zur göttlichen Allwissenheit und Allmacht erscheinen lässt, durchaus verbindlich. ${ }^{\mathrm{i}}$ Weil aber die Problematik der archaischen Anthropologie in B 6 direkt mit der Wegeproblematik

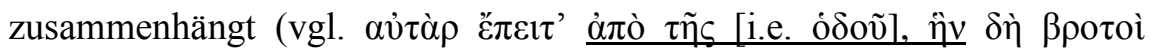

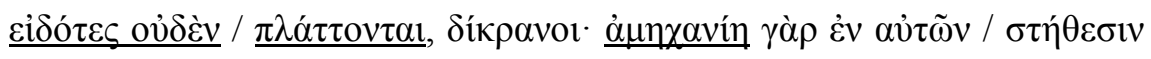

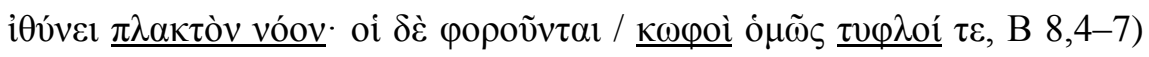
und weil im Gedicht das Motiv zweier Wege entfaltet zu werden scheint, ist anzunehmen, dass für zwei im Gedicht angeführte Wege die dualistische Weltauffassung der archaischen Anthropologie, in der die Menschen im scharfem Gegensatz zu den Göttern stehen, maßgebend sein muss.

Erst dadurch erhält das Motiv zweier Wege seine tiefe und volle Bedeutung: Der eine (göttliche) Weg hängt mit der Wahrheit (dem unentstandenen und unvergänglichen દ̇óv) zusammen; der andere (menschliche) Weg dagegen mit der menschlichen Welt. Auf diesem Hintergrund ist es klar, dass die Wege nicht Wege eines diskursiven

i Mansfeld, J., Die Offenbarung des Parmenides und die menschliche Welt, Assen 1964, S. $1-41$. 
Denkens sind, wie man oft behauptet, ${ }^{\mathrm{i}}$ sondern vielmehr zwei grundverschiedene Lebensweisen darstellen; die eine entspricht dem Leben der allwissenden und allmächtigen Götter, während die andere das jämmerliche Leben der Menschen darstellt. Die Wege sind also Lebenswege (vgl. auch Abschnitt III. 5.).

\section{III.1 Das Fragment B 2}

Nachdem wir Parmenides' Konzeption von éóv behandelt und neue Struktur des Gedichts angedeutet haben, versuchen wir noch die genaue Gestalt des zweiten Wegeteils zu entwerfen und einige mit ihm zusammenhängende und für das Verständnis des Gedichts wichtige Probleme und Gedanken zu interpretieren. Den Anfang des zweiten Teils, den der Abschluss von B 1 bildet und in dem das Motiv zweier Wege angegeben wird, haben wir schon genügend erläutert. Deshalb können wir auf das wichtige Fragment B 2 eingehen.

Bei der Interpretation von B 2 gibt es hauptsächlich zwei

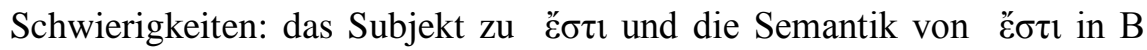

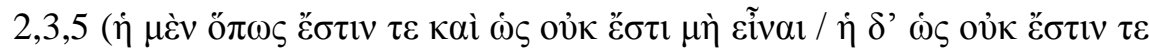

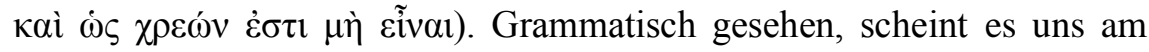

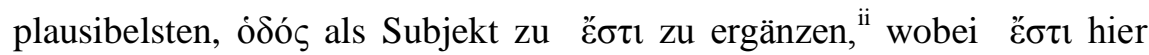

\footnotetext{
i Vgl. z.B. Fritz, K. von., „Die Rolle des nous“, in: H.-G. Gadamer (ed.), Um die Begriffswelt der Vorsokratiker, Darmstadt 1989, S. 246-363, S. 314.

ii Vgl. Kahn, Ch. H., „The Thesis of Parmenides“, op. cit., S. 709: "But the verb है $\sigma \tau$ is not normally used in Greek as an impersonal in the sense just described. It generally occurs either with a grammatical subject or with a logical subject easily identified from the context."
} 
zuerst in der veritativen Bedeutung aufzufassen ist. (Es ist aber gleich

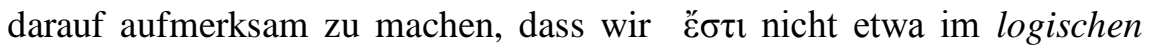
Sinn von Kahns These, sondern ganz allgemein als stilistisches Mittel, etwas als wahr zu betonen, verstehen - es soll also nach dem geläufigen Ausdruck ह̌ $\sigma \tau \imath \tau \alpha \tilde{v} \tau \alpha$ gebildet sein.) Eine solche Charakterisierung beider Wege als wahr resp. unwahr in B 2,3,5 (,Der eine Weg, dass er (wahr) ist und dass es unmöglich ist, dass er nicht (wahr) ist; der andere, dass er nicht (wahr) ist und dass es nötig ist, dass er nicht (wahr) ist.") bereitet auf dem Hintergrund der archaischen Anthropologie keine Schwierigkeiten. Denn in dieser Interpretation wollen beide Verse sagen, dass der eine (göttliche) Weg wahr sein muss, während der andere (menschliche) aus dem Gesichtspunkt des ersten Wegs als unwahr erscheinen muss.

Obwohl diese Interpretation plausibel wirkt, reicht sie dennoch nicht aus. (Nun gilt es, sich noch einmal die Tatsache zu vergegenwärtigen, dass wir das Gedicht interpretieren. Durch das ontologische Verständnis ist man verpflichtet, im Text des Parmenides nach einer logisch präzisen und eindeutigen Argumentation zu suchen; für ein Gedicht ist es dagegen nicht nur charakteristisch, sondern auch konstitutiv, dass es mit Mehrdeutigkeiten, Assoziationen, Evozierungen und überhaupt mit Strategien, die nicht argumentativ verfahren, arbeitet.) Wenn Parmenides nur über die Wahrheit bzw. Unwahrheit der Wege sprechen wollte, würde er sich, um sie zu charakterisieren, einfach der

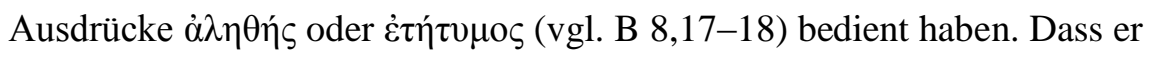
sie aber mit Hilfe von drei hintereinander vorkommenden દ̌ $\sigma \tau \imath$ resp.

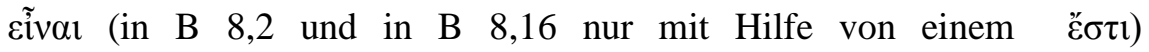
charakterisiert, muss einen guten Grund gehabt haben. Die beiden Verse 


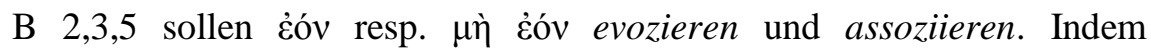
Parmenides zur Charakterisierung beider Wege als wahr resp. unwahr eine so effektive dichterische Figur (B 2,3,5) verwendet, die fähig ist,

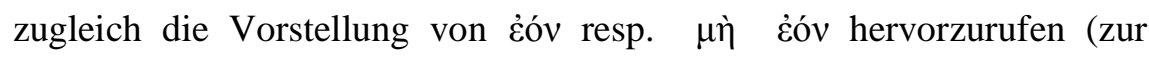

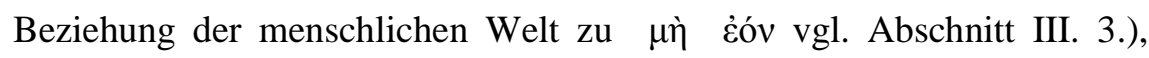
sichert er die notwendige Verbindung zwischen den beiden Wegen und

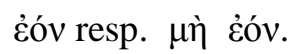

Interpretatorisch lassen sich also in beiden Versen zwei Ebenen unterscheiden. Auf der einen Ebene ist als Subjekt zu Ě aufzufassen, und was die Semantik von Eĩval angeht, ist zuerst aus der breiten Skala der verschiedenen Aspekte von Eivval der veritative Aspekt hervorzuheben. (Dadurch werden beide Wege als wahr resp. unwahr charakterisiert.) Auf der anderen Ebene streben die Äußerungen in B

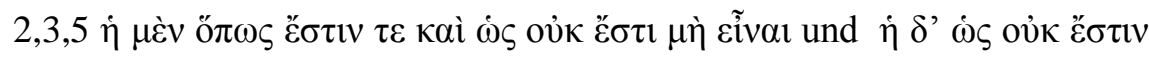

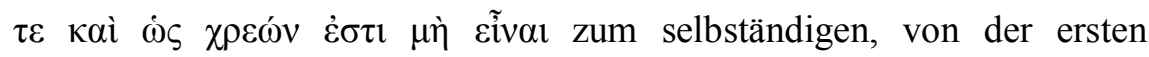
(,grammatischen“) Ebene unabhängigen Ausdruck. (Dadurch wird der „Inhalt" beider Wege oder das auf ihnen zu begegnende Objekt evoziert und so als notwendig mit beiden Wegen verbunden.) - Es ist ersichtlich, dass beide Verse zu komplex sind, um als bloße Glieder einer ontologischen Argumentation abgetan zu werden. Sie sollten zuletzt in ihrer dichterisch-denkerischen Fülle und Tiefe genossen werden. 


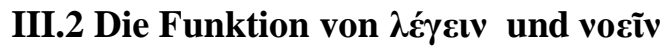

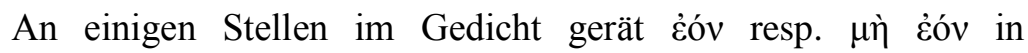

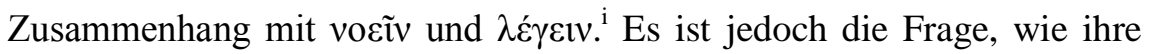
Beziehung genau zu verstehen ist. Bedenken wir, dass vocĩv anhand des Modells der Sinneswahrnehmung ${ }^{\text {ii }}$ verstanden werden muss, dann kann

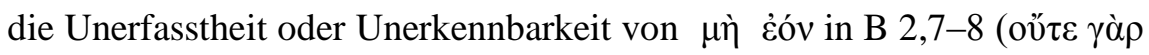

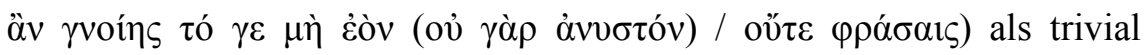
gelten $^{\text {iii }}$ : was nicht ist, kann nicht erfasst oder erkannt werden. Warum ist aber $\mu$ ì દ̇óv unsagbar? Man kann seine Unsagbarkeit als Unsagbarkeit eines Objekts deuten, indem man annimmt, dass $\varphi \rho \alpha ́ \zeta \varepsilon ı v ~ r e s p . ~ \lambda \varepsilon ́ \gamma \varepsilon ı v$ nach der Struktur von voeĩv, also nach dem Modell des Erfassens (Erkennens) von etwas gebildet ist. ${ }^{\text {iv }}$ Eine solche Verschmelzung der semantischen Struktur der beiden Termini leuchtet aber nicht ein: Denn es ist keineswegs unproblematisch zu behaupten, dass ein Objekt unsagbar sei - und zwar schon deshalb, weil die Richtigkeit einer solchen Behauptung verneint wird, wenn man es einfach ausspricht. Parmenides scheint deshalb mit $\varphi \rho a ́ \zeta \varepsilon ı v$ resp. $\lambda \varepsilon ́ \gamma \varepsilon ı v$ etwas anderes zu meinen.

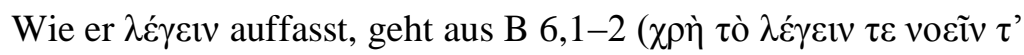

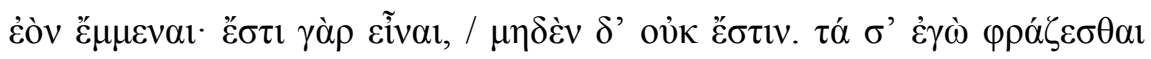

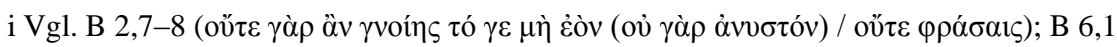

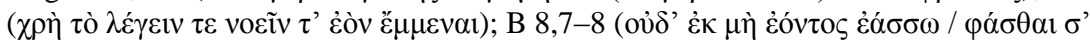

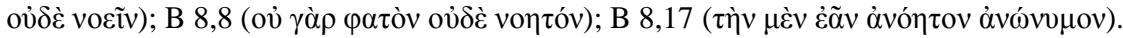
ii Vgl. Heitsch, E., Parmenides. Die Anfänge der Ontologie, Logik und Naturwissenschaft, op. cit., S. 99-100. Fritz, K. von., „Die Rolle des nous“, op. cit.

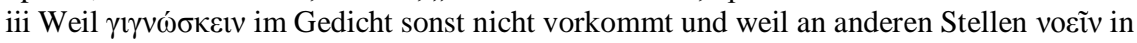

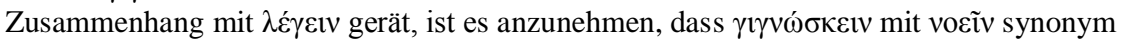
ist.

iv Vgl. Tugendhat, E., „Das Sein und das Nichts“, op. cit., S. 45-46. Wiesner, J.,

Parmenides. Der Beginn der Aletheia, op. cit., S. 25-27. 
ăv $\omega \gamma \alpha)$, das sich ursprünglich in der Nähe von B 2 befunden haben soll, ${ }^{\mathrm{i}}$

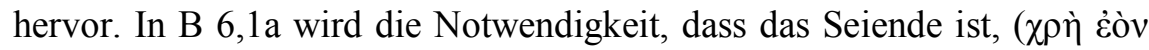

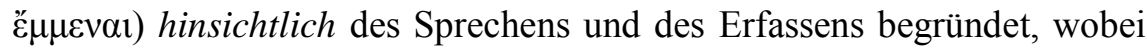

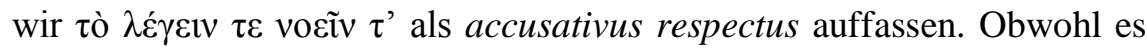
uns heute ganz und gar verwundern kann, dass jemand die ontologische oder faktische Notwendigkeit sprachlich zu begründen sucht, scheint Parmenides eben dies gemeint zu haben.

Die beiden, aus drei Sätzen bestehenden Verse B 6,1-2 haben also folgende argumentative Struktur. Zuerst wird in B 6,1a die Notwendigkeit dessen, dass das Seiende ist, hinsichtlich der Sprache und des Erfassens behauptet. Während - wie wir gesehen haben - die Begründung anhand des Erfassens ohne weiteres klar ist und keiner zusätzlichen Erklärung bedarf, erklärt Parmenides in den folgenden zwei Sätzen, wie die sprachliche Begründung aufzufassen ist. Er unterstützt sie (vgl. die begründende Partikel $\gamma \alpha \dot{\alpha} \rho$ in B 6,1b) zuerst durch seine im zweiten Satz ausgedrückte These oder Maxime, dass das Seiende ist, das Nichts aber nicht ist ( Dass eben diese These die sprachliche Begründung der (ontologischen)

\footnotetext{
i Es wird manchmal sogar vorausgesetzt, dass B 2, B 3 und B 6 unmittelbar hintereinander folgen und in ihnen eine kontinuierliche ontologische Argumentation zu finden ist.

Hinsichtlich der bruchstückhaften Überlieferung des Gedichts wirkt aber die unmittelbare Nachfolge dieser Fragmente gewissermaßen künstlich. Dabei ist noch zu erwägen, ob B 3 überhaupt echt ist und nicht eine spätere Paraphrase des in B 8,34 enthaltenen Gedankens darstellt. Wir neigen zu dieser Vermutung - und zwar um so mehr, da wir nicht einmal in B 8,34 die Identität vom Sein und Denken ausgedrückt sehen; denn $\tau \alpha$ vóóv ist hier u. E. ebenso wie in B 8,29 mit દ̇óv gleichwertig; $\tau \alpha$ vóv heißt hier also die Identität mit sich selbst und nicht die Identität zwischen zwei Sachen. Ähnlich Wiesner, J., Parmenides. Der Beginn der Aletheia, op. cit., S. 151. „Wie ich in einer früheren Arbeit ausgeführt habe, nennt B 8,34 zwei Voraussetzungen für diese Erkennbarkeit des Seienden: ,als Identisches kann es erkannt werden und weil die Erkenntnis Bestand hat'." Zu diesem Vers vgl. auch Abschnitt III. 5.
} 
Notwendigkeit dessen, dass das Seiende ist, liefert, belegt der dritte Satz (B 6,2b), wo die Göttin ihren Zuhörer auffordert (ő $\alpha \omega \gamma \alpha$ ), auf diese Weise resp. (wortwörtlich) diese Sachen ( $\tau \alpha$ ), die die These enthält, zu sich zu sprechen ( $\varphi \rho \alpha ́ \zeta \varepsilon \sigma \theta \alpha \mathrm{l})$. In anderen Worten, die These, dass das Seiende ist, das Nichts aber nicht ist, kann nicht bloß als Konstatierung eines Sachverhalts gedeutet werden, sondern ihre innere sprachliche Logik, nach der es unmöglich wäre, anders $\mathrm{zu}$ sprechen, ist vor allem zu berücksichtigen. Parmenides' Auffassung der Sprache war also grundverschieden von dem, wie wir die Sprache und ihre Beziehung zur Realität auffassen. Ihm war die Sprache nicht ein bloßes, von der Wahrheit oder Wirklichkeit losgelöstes Werkzeug, sondern vielmehr wie für die ganze damalige archaische Zeit - eine elementare Macht, deren Wahrheit keinen Widerspruch von der Art, dass das, was nicht ist ( $\mu$ ì દóv), ist, zuließe. Zwischen der Sprache und der Wirklichkeit gibt es also bei Parmenides keine unüberbrückbare Kluft - beides stellt höchstens zwei verschiedene Aspekte des Gleichen dar. (Die ganze Übersetzung von B 6,1-2 lautet: „Hinsichtlich des Sprechens und Erfassens ist es notwendig, dass das Seiende ist. ${ }^{i}$ Denn das Seiende ist, das Nichts ist aber nicht. Ich fordere dich auf, auf diese Weise (diese Sachen) zu sich zu sprechen.")

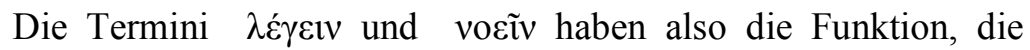
Notwendigkeit von દ́óv resp. die Unmöglichkeit von $\mu$ そ̀ દ̇óv zu sichern. $\lambda \varepsilon ́ \gamma \varepsilon ı v$ tut dies im Hinblick auf das richtige Funktionieren der Sprache,

\footnotetext{
i Als Subjekt in B 6,1b ist das in B 6,1a vorkommende çóv zu ergänzen. Was die

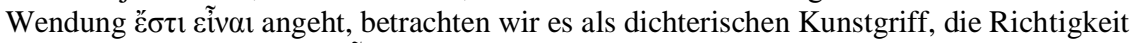

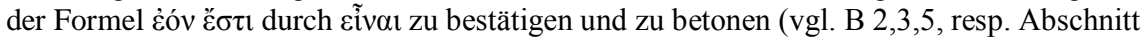
III.1.).
} 
voeĩv anhand des intuitiven Erfassens. Ihre begründende Funktion erfolgt vornehmlich aufgrund ihres elementaren und wahrhaftigen Charakters. Sie begründen also unmittelbar aus sich selbst und nicht - wie man gewöhnlich annimmt - erst mittelbar dadurch, dass sie als bloße Glieder oder Prämissen in einer Argumentation auftreten. Das dichterische Element von Parmenides' Gedicht fordert ja, nicht alles im Text logisch oder begrifflich zu entzaubern, sondern es in seiner ursprünglichen elementare und mythischen Fülle und Tiefe zu verstehen zu suchen.

Durch B 6,1-2 erhält auch die Charakterisierung beider Wege noch eine andere wichtige Dimension. Der erste Weg wird mit der (Notwendigkeit der) Äußerung „Das Seiende ist, das Nichtseiende ist nicht“ verknüpft, während der zweite die (Unmöglichkeit der) Äußerungen wie „Das Nichtseiende ist“ (vgl. B 7,1) oder „Das Seiende ist nicht" (die ja in Widerspruch zum richtigen Funktionieren von $\lambda \varepsilon ́ \gamma \varepsilon t v$ stehen) impliziert.

\section{III.3 Zweifache Perspektive der menschlichen Welt}

Nachdem also das Motiv zweier Wege am Ende von B 1 angegeben und in B 2 und B 6,1-2 entwickelt und ausgearbeitet wird, geht Parmenides auf eine gründlichere Behandlung des zweiten Wegs ein. Doch bevor wir versuchen, sie zu rekonstruieren und interpretieren, sollen wir das folgende Problem erwägen: Die von B 8,53 (also im vierten Teil) beschriebene menschliche Welt, die ja der „Inhalt“ des zweiten Wegs sein soll, wird als Mischung zweier elementarer Kräfte von Tag und Nacht aufgefasst (B 8,53-59). Dabei ist es gleich darauf aufmerksam zu machen, dass diese Konzeption der Welt das Nichtseiende ausschließt 


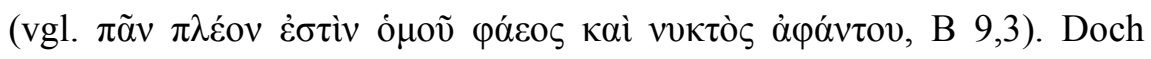
haben wir mehrmals (hauptsächlich im Zusammenhang mit dem, was im zweiten Wegeteil gesagt wird) festgestellt, dass der zweite Weg das Nichtseiende voraussetzt und impliziert. Das Gedicht scheint also zwei Auffassungen der menschlichen Welt zu implizieren: Während im zweiten Wegeteil die menschliche Welt unter der Perspektive des Nichtseienden betrachtet wird, entwickelt der vierte Teil die Auffassung der Welt als der Mischung zweier elementarer Grundelemente, Licht und Nacht, die keinen Raum für das Nichtseiende zulassen.

Für die Lösung dieser Schwierigkeit ist die richtige Rekonstruktion des Restes des zweiten Wegeteils, der sich von B 6,4 über B 4 bis zu B 7 weiter erstreckt, von großer Bedeutung. Die entscheidende

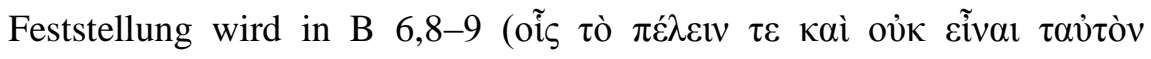

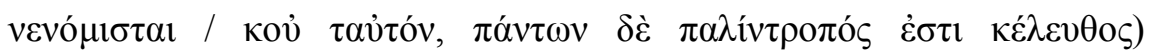
angegeben: die Menschen vermögen nicht zwischen ,sein ‘ und ,nicht sein' resp. zwischen den Dingen, die sind, und jenen, die nicht sind, zu unterscheiden (die substantivierten Infinitive sind nicht mit żóv resp. $\mu \grave{\eta}$ ćóv gleichzusetzen, sondern sie behalten vielmehr die verbale Funktion).

Was es genau heißt, dass die Menschen die seienden und nichtseienden Dinge vermischen, erhält eine präzisere Bedeutung durch die weitere Fortsetzung des Wegeteils in B 4 und B $7^{\mathrm{i}}$, wo zuerst über die

i Vgl. Wiesner, J., Parmenides. Der Beginn der Aletheia, op. cit., S. 237-250, vor allem 246. ,Wir können nun den nach B 6,9 anschließenden Gedankengang klar rekonstruieren: $<$ Die irrenden Menschen, die fälschlich ,sein' und, nicht sein“ gleichen, stufen den Sinnen Abwesendes ( $\dot{\alpha} \pi \varepsilon o ́ v \tau \alpha)$ als Nichtseiendes ein.> Doch du schau Abwesendes mit dem geistigen Auge beständig als Anwesendes. Denn der Nus wird das Seiende nicht davon abtrennen, mit dem Seienden zusammenzuhängen etc. (B4). $<$ Er wird somit Abwesendes richtig als Seiendes, das es ist, erfassen und nicht nach der menschlichen Weise als 


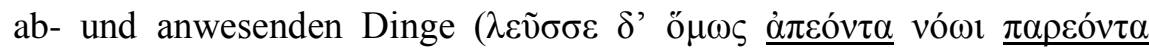

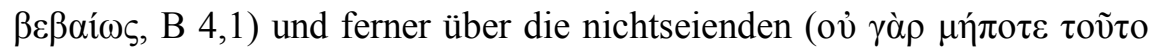

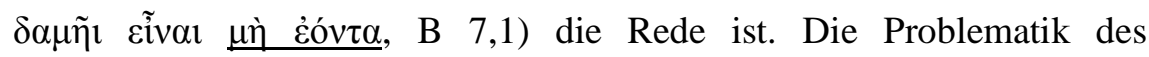

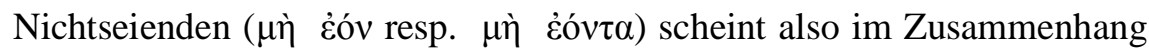
mit der Ab- und Anwesenheit gedacht werden. In der Tat erfahren wir in unserer Lebenswelt infolge der Sinneswahrnehmung einige Dinge als abwesend. Und weil diese $\alpha \dot{\tau} \varepsilon$ óv $\tau \alpha$ nicht zu sein scheinen, lässt sich über

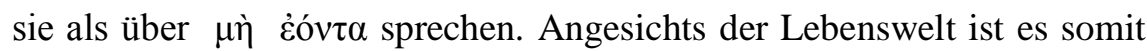

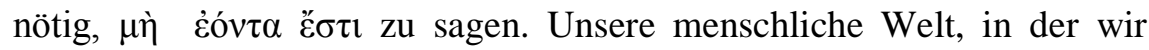
leben, setzt also (wegen der Sinneswahrnehmung) unumgänglich die

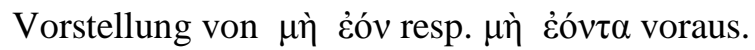

Der scheinbare Widerspruch beider Konzeptionen der menschlichen Welt ist also durch die Schlüsselrolle des damals für die

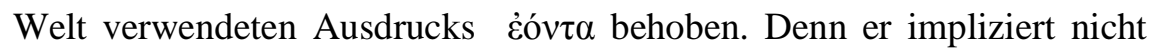
nur die Pluralität der Seienden, die die (triviale, zugleich aber fundamentale) Voraussetzung der menschlichen Welt ist, sondern auch das Nichtseiende, das wiederum eine Konsequenz der (Sinneswahrnehmung der) Pluralität ist. Obwohl es in Parmenides' Gedicht im Grunde nur eine einzige, auf ćóv $\tau \alpha$ gründende Konzeption der menschlichen Welt gibt, kann sie jedoch aus zwei verschiedenen, auf eine je andere Wirkung abzielenden Perspektiven betrachtet und inszeniert werden. In dem zweiten Wegeteil verwendet (inszeniert) Parmenides die mit dem Nichtseienden zusammenhängende Auffassung der menschlichen Welt zur Herabsetzung und Verwerfung des zweiten Wegs, was zugleich den Vorzug, ja die Notwendigkeit des ersten Wegs

Nichtseiendes einstufen.> Denn es kann niemals erzwungen werden, dass Nichtseiendes

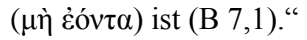


gegenüber dem zweiten zeigen soll. Denn obwohl es in unserer menschlichen Welt einen guten Sinn hat, über das Nichtseiende resp. darüber, dass das Nichtseiende ist, zu sprechen, ist es anhand des richtigen, ja

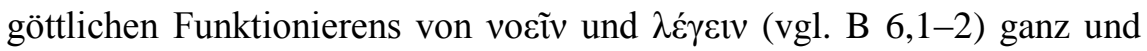

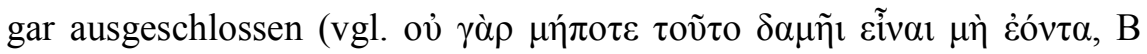
7,1). Weil diese Auffassung der Welt anhand der Sinneswahrnehmung, die ja die Abwesenheit und so das Nichtseiende impliziert, konstituiert wird, könnte man sie als perzeptorische Konzeption der Welt bezeichnen. Weil dagegen die andere, in dem vierten Teil entwickelte Konzeption der Welt aus der Sicht der Göttin vorgetragen and als Belehrung über die menschliche Welt gemeint wird, könnte sie als eine Art wissenschaftlicher Konzeption der Welt begriffen werden. Aus der göttlichen Perspektive des ersten Wegs muss jedoch zuletzt auch diese wissenschaftliche Konzeption als unzulänglich, ja trügerisch erscheinen -

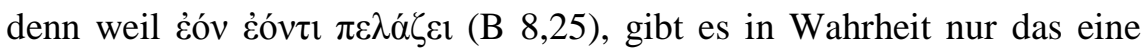
غ̇óv.

\section{III.4 Die Beziehung zwischen ċóv und der menschlichen Welt}

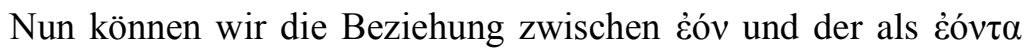
aufgefassten menschlichen Welt anhand von B 4 genau bestimmen. Der Unterschied zwischen ihnen ist letztlich kein essentieller (in dem Sinne, dass zwischen ihnen eine Wesen- oder Grundverschiedenheit besteht bzw. dass sie zwei verschiedene Seinsregionen des Ganzen darstellen), sondern nur ein perspektivischer. Denn er hängt mit vóos zusammen. Wie

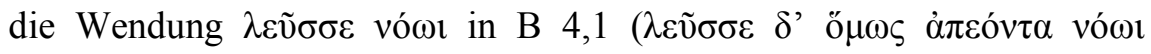

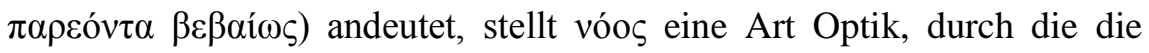


Welt anzusehen ist, dar. Wenn man also durch vóos die abwesenden Dinge als anwesend ansehen soll und wenn - wie wir gesehen haben - die abwesenden als nichtseiend gelten, dann ist es vóos, von dem es abhängig

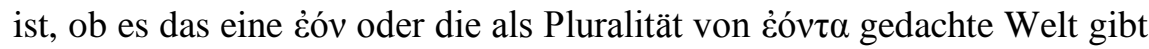
resp. - damit wir der perspektivischen Hinsicht Rechnung tragen - ob

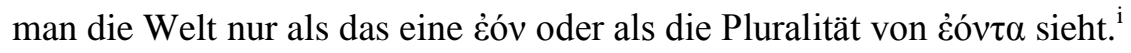

i In unserem Artikel behandeln wir die Problematik der Beziehung zwischen żóv und der menschlichen Welt vor allem aus der (strukturellen) Perspektive zweier Wege. Versuchen wir aber diese Problematik ein bisschen allgemeiner aufzufassen. Wenn im Gedicht ćóv und die menschliche Welt behandelt werden, scheint Parmenides' Konzeption irgendwie dualistisch zu sein. Das widerspricht jedoch seinen vor allem in B 8 vorkommenden Behauptungen, dass es nur das eine ćóv gibt. Wenn man ihn aber wiederum für einen Monisten halten will, muss man über seine Auffassung der menschlichen Welt irgendwie hinweggehen und ihre Möglichkeit ganz bestreiten. In anderen Worten, obgleich Parmenides' Position in B 8 ganz monistisch aussehen kann, ist sie nur schwerlich mit seiner durchgehenden Anstrengung, die menschliche Welt zu denken und zu thematisieren, vereinbar. Der Vorteil unserer perspektivischen Auffassung der Beziehung zwischen ćóv und der menschlichen Welt besteht darin, dass sie das angebliche monistisch-dualistische Dilemma gewissermaßen aufhebt. Unsere Interpretation ist nämlich sowohl monistich als auch dualistisch. Sie ist - ontologisch gesagt - insofern monistisch, als es nur ein Etwas, das auf zwei verschiedene Weisen betrachtet wird, gibt. Doch - perspektivisch betrachtet - ist sie dualistisch, denn das eine éóv von zwei verschiedenen Perspektiven aus betrachtet werden kann. Nun, man könnte sich fragen, wie dieses eine Etwas, das zweierlei betrachtet werden kann, aufzufassen ist. Es war Parmenides' Absicht zu zeigen, dass es in Wahrheit nur das eine ċóv gibt, und dass unsere

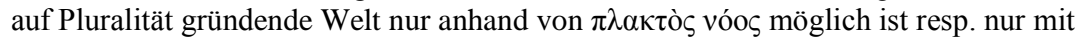
unserer falschen Benennung zusammenhängt (vgl. B 8,38-41). Deshalb könnte man einfach meinen, dass das eine Etwas, das zweierlei betrachtet werden kann, einfach das eine ćóv sein muss und dass die menschliche Welt gar keine reale (ontologische) Wirklichkeit haben kann (weil es sich ja - wie es im Gedicht heißt - bloß um eine falsche Benennung handelt). Wie aber unsere Ausführungen andeuten, ist die Problematik der menschlichen Welt in Parmenides' Gedicht nicht so einfach und eindeutig. Die Absicht oder Intention des Autors ist eine Sache, eine ganz andere ist es aber, wie der Autor zu seiner Einsicht gekommen ist und wie er sie in seinem Text ausarbeitet und so seinem Publikum präsentiert. Parmenides spricht zwar so, als ob das eine ćóv das einzig Existierende wäre. Doch seine ganze denkerische Konzeption (d.h. das denkerische Gerüst, um das herum das Gedicht aufgebaut wird) beruht darauf, dass es die als żóv $\tau \alpha$ gedachte Welt ist, was es gibt und was wir zweierlei betrachten können. In anderen 
Dass es also غ̇óv gibt resp. dass wir غ̇óv sehen und begegnen, hängt von vóoৎ ab. Man könnte sich aber fragen, ob man wirklich auch die Welt durch vóos ansehen kann, denn spätestens seit Platons Ablösung der immateriellen Ideen von der materiellen Welt sind wir daran gewöhnt, die Tätigkeit von (dem als Denken oder Geist verstanden) vóo ̧ mit dem Immateriellen oder Geistigen zu verbinden und die Welt mittels der Sinneswahrnehmung zu erfassen. Bei der Interpretation eines archaischen Denkers müssen wir aber alle platonischen Denkschemata ablegen. Und wie der Imperativ $\lambda \varepsilon \tilde{\sigma} \sigma \sigma \varepsilon$ in B 4,1 andeutet, kann die Wirksamkeit von vóo Welt einschließen. Wenn vóoৎ nur mit દ̇óv irgendwie wesensverwandt wäre, hätte der Imperativ keinen Sinn. ${ }^{\mathrm{i}}$

Dass sich vóo $\varsigma$ auch auf die Welt bezieht, wird auch im Fragment B 6 bestätigt, wo er explizit mit dem zweiten Weg zusammenhängt und wo er überdies mit dem Prädikat $\pi \lambda \alpha \kappa \tau$ ó (,schweifend“) versehen ist. Wenn nun vóos ein mit dem zweiten Weg zusammenhängender $\pi \lambda \alpha \kappa \tau$ ò $\varsigma$ vóos (B 6,6) ist, der ,sein“ mit ,nicht sein‘ vermischt (vgl. B 6,8-9), sieht man nicht nur einige Dinge als abwesend, also nichtseiend (vgl. die perzeptorische Konzeption der Welt innerhalb des zweiten Wegeteils), sondern auch ihre Mannigfaltigkeit (vgl. die ab B 8,53, also im vierten Teil entwickelten wissenschaftliche Konzeption der Welt); anders gesagt,

Worten, die Problematisierung, ja Ablehnung der Welt zugunsten des einzigen éóv ist vielmehr eine rhetorische oder dichterische Figur, die kontextuell gewertet werden muss, und nicht eine strikte denkerische Angelegenheit. Das Problem der meisten interpretatorischen Versuche über Parmenides liegt nebenbei eben darin, dass sie Parmenides' Gedicht zu wortwörtlich nehmen, ohne sich seine denkerische Konzeption anzueignen.

i $\mathrm{Zu}$ der Identität von ,Denken“ und Sinneswahrnehmung in Parmenides vgl. auch

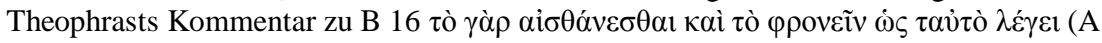
46). 
auf diesem zweiten Weg begegnet man die Welt. Wenn man sich dagegen auf dem ersten Weg befindet und den richtig funktionierenden und ansehenden vóos hat, sieht man alle Dinge als anwesend, also seiend; und weil vóos nicht દ̇óv von ćóv abschneidet (B 4,2), begegnet man nur das eine unentstandene und unteilbare éóv (vgl. auch B 8,22-25).

Nun, die Verbindung von vóo $̧$ mit beiden Wegen ist eines der prinzipiellsten Momente für das Verständnis des Gedichts. Denn der Unterschied zwischen દ̇óv und der Welt liegt letztlich darin, auf welchem Weg man sich befindet resp. aus welchem Weg man die Welt ansieht. Und weil die Wege als Lebenswege zu verstehen sind, hängt es letztlich von unserer Lebensweise oder von unserem Charakter (zur praktischen Bedeutung von vóos als Charakter vgl. Abschnitt IV.) ab, ob wir ćóv oder die menschliche pluralistische Welt begegnen.

\section{Schluss: Praktisch-religiöser Charakter des Gedichts}

Obwohl man Parmenides' Gedicht gewöhnlich als einen sich nur mit theoretisch-ontologischen Fragen auseinandersetzenden Traktat betrachtet, tritt nun (besonders auf dem Hintergrund der mit zwei Wegen zusammenhängenden archaischen Anthropologie) sein praktischreligiöser Charakter unübersehbar hervor. Nicht nur dass auf dem Hintergrund der archaischen Anthropologie die Wege als Lebenswege gedacht werden müssen (vgl. Abschnitt II.2), sondern sie erhalten ihren praktischen Charakter auch dadurch, dass sie - wie wir im letzten Abschnitt gesehen haben - den je verschiedenen vóo $\varsigma$ als die für jeden Weg charakteristische Sichtweise der Welt implizieren. 
Der praktische Charakter des Gedichts manifestiert sich auch in der Konzeption von vóo $\varsigma$ selbst. Denn vóoৎ darf nicht nur als eine rein theoretische (sei es denkende, sei es betrachtende) Fähigkeit aufgefasst werden. Wie auch seine Verwendung im damaligen Kontext belegt, hängt er mit dem menschlichen Gemüt oder Charakter eng zusammen, was noch damit betont wird, dass er oft mit moralisch wertenden Attributen vorkommt. ${ }^{i}$ Was seine Verwendung in Parmenides' Gedicht angeht, ist diese praktische Auffassung von vóos durch sein innerhalb der archaischen Anthropologie als moralisch $\mathrm{zu}$ fassende Attribut $\pi \lambda \alpha \kappa \tau$ ó $\varsigma$ ziemlich eindeutig bestätigt. Denn innerhalb der archaischen Anthropologie wird die in B 6,6 vorkommende Wendung $\pi \lambda \alpha \kappa \tau$ ò $\varsigma$ vóos nicht nur im theoretischen Sinn (etwa als fehlende Sichtweise/Vernunft), sondern als schweifender oder gar labiler Charakter verstanden.

Wenn wir diese praktisch-moralische Färbung von vóos in Betracht ziehen und wenn wir zugleich erwägen, dass seine Nähe zu der Wegeproblematik seinen praktischen Charakter im Gedicht unterstreicht (vgl. B 6), dann darf es nicht verwundern, dass auch der mit vóos

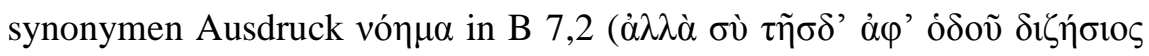

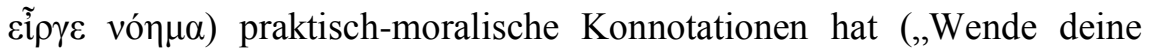
Sichtweise der Welt/deinen Charakter vom diesem Weg der

i Wenn man sich im Kontext des Gedichts von Parmenides umsieht, wird man feststellen müssen, dass vóos hier oft soviel wie Charakter oder Gemüt heißt - was noch durch die

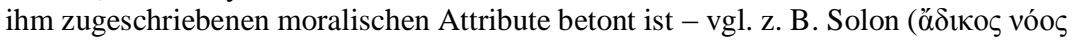

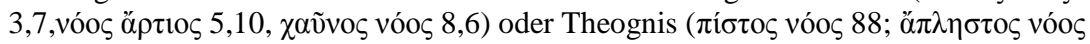

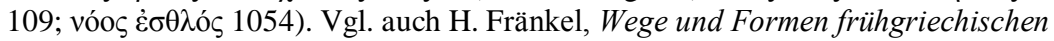
Denkens, München 1955, S. 28. Anm. 3: „Der Begriff, Charakter ${ }^{6}$ jedoch war noch nicht klar entwickelt; die dafür hier gebrauchten Worte sind bei Homer vóo $\varsigma$ und bei Archilochos $\theta v \mu$ ó $\varsigma$ und $\varphi \rho o v \varepsilon ́ \varepsilon v v$. Sowohl vóo $\varsigma$ als $\theta v \mu o ́ \varsigma$ kann bedeuten ,Geist, Gemütsverfassung, Haltung، ““ 
Untersuchung“"). Denn wenn man sich auf dem ersten Weg befindet, muss man nicht nur der für den zweiten Weg charakteristischen Sichtweise loswerden, sondern es ist notwendig, sich auch einer innerlichen Verwandlung zu unterziehen. Ähnlich behandelt auch der bekannte Vers

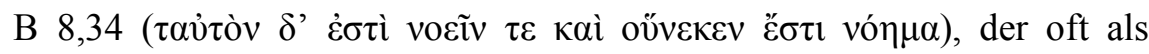
Identität vom Sein und Denken interpretiert wird, nicht nur einen theoretischen Bezug zwischen ćóv und vó $\mu \alpha$, sondern er ist auch so zu lesen, dass nur éóv als etwas Göttliches den Menschen den unerschütterlichen Charakter, der im Gegensatz zu ihrem schweifenden Wesen steht, gewährt. ${ }^{\mathrm{i}}$

Der praktisch-religiöse Charakter macht sich auch in der bekann-

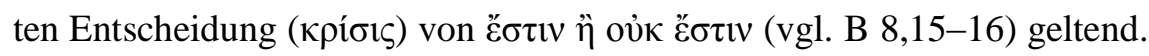
Man kann sie also nicht - wie man es gewöhnlich tut - als das logische Gesetz des ausgeschlossenen Dritten auffassen. Es handelt sich in ihr

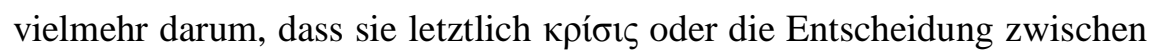
den beiden Wegen ist. Der erste Weg kann ja durch દ̌ $\sigma \tau \iota v$ charakterisiert

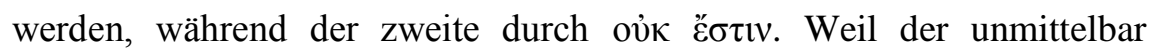
vorhergehende Teil von B 8 die Unmöglichkeit des Entstehens und Vergehens von ćóv behandelt, ist die Entscheidung zwischen den beiden Wegen letztlich als Entscheidung darüber, ob es das Entstehen und Vergehen überhaupt gibt, zu verstehen. In anderen Worten, wenn man

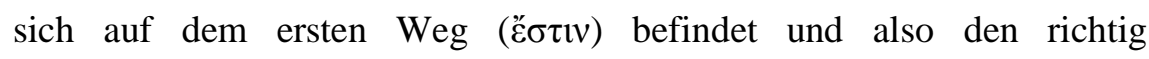
funktioneirenden vóo $\varsigma$ besitzt, wird man überall nur das eine čóv, in dem für das Entstehen und Vergehen kein Platz ist, begegnen. Wenn man sich dagegen auf dem zweiten, mit $\pi \lambda \alpha \kappa \tau$ ò $\varsigma$ vóo $\varsigma$ zusammenhängenden Weg

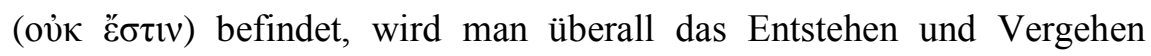

i Zur Interpretation von B 8,34 vgl. meine Studie XXX. 
begegnen; „es wurde jedoch mit der Notwendigkeit entschieden (in dem zweiten, anhand der Konfrontation beider Wege inszenierten Teil des Gedichts), dass man sich vom zweiten Weg abwenden soll, weil nur der

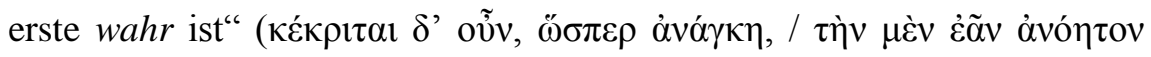

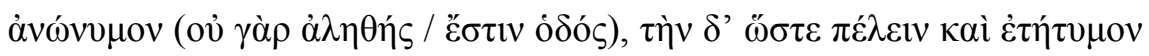
ยิ๊ขa1, B 8,16-18).

Nun, obwohl der praktisch-religiöse Charakter mehrere, ja fast alle Motive und Gedanken des Gedichts durchzieht, ist es die so-genannte archaische Anthropologie, die sie verbindet und ihnen die Rechtfertigung und den Sinn gibt. Nichtsdestoweniger Parmenides' Auffassung der archaischen Anthropologie ist letztlich nicht mit ihrer traditionellen Fassung identisch. Es ist nämlich Parmenides gelungen, sie philosophisch zu überwinden. Denn indem er die archaische Anthropologie anhand der beiden als Lebenswege gedachten Wege perspektiviert, hat er sie ihres essentiellen und unüberbrückbaren Gegensatzes zwischen Menschen und Götter enthoben. Denn während der Mensch in der ursprünglichen Auffassung der archaischen Anthropologie völlig dem Irrtum, der Unwissenheit und Hilflosigkeit den Götter gegenüber preisgegeben wurde, ist die unüberbrückbare Kluft zwischen Menschen und Göttern bei Parmenides durch die zentrale Rolle, die er der auf vóoৎ gründenden Sichtweise zugeschrieben hat, beseitigt; es reicht, wenn vóos richtig funktioniert, d.h. sieht, damit man auf den ersten, göttlichen Weg, auf dem man nur das eine unentstandene und unteilbare ćóv begegnet, gerät. Doch dazu - wie die Offenbarung der Göttin zeigt - ist die göttliche Hilfe immer nötig. 